\title{
Four-month outbreak of invasive meningococcal disease caused by a rare serogroup B strain, identified through the use of molecular PorA subtyping, England, 2013
}

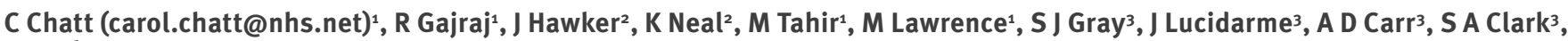
T Fowler ${ }^{2,4}$

1. West Midlands East Health Protection Team, Public Health England, Birmingham, United Kingdom

2. Field Epidemiology Service - West Midlands, Public Health England, Birmingham, United Kingdom

3. Meningococcal Reference Unit, Public Health England, Manchester, United Kingdom

4. Public Health, Epidemiology and Biostatistics, the University of Birmingham, Birmingham, United Kingdom

Chatt C, Gajraj R, Hawker J, Neal K, Tahir M, Lawrence M, Gray SJ, Lucidarme J, Carr AD, Clark SA, Fowler T. Four-month outbreak of invasive meningococcal disease caused by a rare serogroup B strain, identified through the use of molecular PorA subtyping, England, 2013. Euro Surveill. 2014:19(44):pii=20949. Available online:

http://www.eurosurveillance.org/ViewArticle.aspx?Articleld=20949

Article submitted on 30 January 2014/ published on 06 November 2014

Molecular PorA subtyping provides information that increasingly requires the adaptation of standard public health approaches to outbreak management. We report an outbreak of a rare subtype of meningococcal infection not previously identified in the United Kingdom (UK). The outbreak occurred in the Warwickshire area in England between February and June 2013. Molecular subtyping allowed the identification of additional cases, prompting an enhanced public health response that included efforts to identify potential social networks that might benefit from chemoprophylaxis. It also prompted swabbing to define nasopharyngeal carriage in the focal nursery and helped explain the unusual epidemiological pattern. Without subtyping to identify a link, the additional cases would have been managed as sporadic cases in accordance with current UK guidance.

\section{Introduction}

Invasive meningococcal disease (IMD) is a significant cause of mortality and morbidity, with the highest incidence in children under the age of five years. Persistent neurological defects are more common among infants and young children $[1,2]$. In the United States (US), an estimated 2,600 people are affected and 300 die from the disease each year [3]. In 2012, the incidence of probable and confirmed IMD in the US was 0.18 per 100,000 population [4]. In Europe, 3,808 confirmed cases of IMD were reported across 29 countries in 2011 [5]. Across all age groups in England and Wales, there were 766 cases of IMD in the epidemiological year $2011 / 12$ and the average annual incidence of IMD was 2 per $100,000[5,6]$.

In Europe and the Americas, meningococcus serogroup $B$ has been the predominant serogroup, particularly since the introduction of vaccines against serogroup $C$
[1]. Most cases are sporadic $[7,8]$. When one case occurs in a pre-school group, the absolute risk of becoming a case within the next four weeks is low, about 1 in 1,500 for each child at the same institution [9]. However, a review by the European Centre for Disease Prevention and Control (ECDC) of the available evidence suggested a relative risk of 22.3 (95\% confidence interval (CI): 12.1-40.9) [7].

While guidance varies across Europe [7], guidance in the United Kingdom (UK) recommends that prophylactic antibiotics are not given to pre-school groups after a single case [9]. The reasons for this include that any benefit is unclear, that there is the possibility of harm, that antibiotics may cause side effects, provide false reassurance about protection and eradicate naturally immunising strains from the nasopharynx, and that clusters in pre-school groups are rare (about three per year in England and Wales) [9]. Following a second case in an educational setting, the risk of a third case in that setting may be as high as $30-50 \%[10,11]$. In UK guidance, a cluster in a single educational institution is defined as 'two or more cases of meningococcal disease occurring in the same pre-school group [or other educational setting] ... within a four week period' [9]. If the two cases are, or could be, caused by the same serogroup, staff and children would normally be offered prophylaxis [9]. If the notification of invasive meningococcal disease is delayed, close contacts should be offered chemoprophylaxis up to four weeks after onset of illness [9]. ECDC guidance is focused on sporadic cases and does not address responses to clusters [7].

Swabbing is not recommended in the UK during outbreaks because decisions have to be taken before the results are available and because rates of carriage 
often bear no relationship to the risk of additional cases [9].

We describe here an outbreak of five cases of meningococcal serogroup $B$ disease that occurred in the Warwickshire area in the UK between February and June 2013. It was unusual in that it did not meet the UK standard definition of an outbreak in an educational setting [9] because it included three cases in a nursery over a four-month period. Others have reported a cluster of cases over a long timescale, e.g. Acheson et al. [12], but this was within a single nuclear family, a setting where there is a higher risk of secondary cases. The authors concluded that it was likely that the strain was reintroduced by a regular non-household contact or unidentified close contact [12]. In addition, Mandal et al. reported an outbreak of 13 cases between January 2008 and November 2010 [13].

In our situation, the cases were only identified as linked through initial serological phenotyping, followed by molecular PorA subtyping undertaken by the national meningococcal reference laboratory. Sequence-based typing is increasingly used as a tool to help outbreak investigation. For instance, whole genome sequencing (WGS) of isolates between 1990 and 2012 showed that WGS has the potential to inform the epidemiological investigation of outbreaks of verocytotoxin-producing Escherichia coli. The WGS results prompted further investigation, which in some cases identified previously obscure links between cases [14].

In this report, our aim is to describe how the information provided by the molecular PorA sequence typing informed and changed our standard response. Our focus is the health protection response to the outbreak and in particular, the challenge of interpreting and acting upon additional microbiological information in this setting. All standard public health actions relating to single cases, such as chemoprophylaxis for household contacts, were undertaken at the time of notification.

\section{Outbreak description}

During February 2013 a two year-old child (Case 1) attending nursery (Nursery 1), was admitted to hospital with symptoms of lethargy, reduced consciousness and a non-blanching rash. The following day, the health protection team was notified of this probable case [9] of meningococcal disease. Three days after the child was admitted, the reference laboratory reported that meningococcus serogroup $B$ had been isolated from the blood culture (in addition to PCR-positive EDTA blood samples) and 7 days later, the organism was confirmed as Neisseria meningitidis phenotypically described as group B, PorB serotype 4, and PorA serosubtype (NT / NT / P1.6). DNA sequencing was used to define the PorA subtype as $\mathrm{P}_{1.18-4}, 25,38-1$. The isolate had reduced susceptibility (intermediate) to penicillin (minimum inhibitory concentration $(\mathrm{MIC})=0.06$ $\mathrm{mg} / \mathrm{L}$ ) but was susceptible to cefotaxime (MIC $=0.002$ $\mathrm{mg} / \mathrm{L})$, rifampicin (MIC $=0.004 \mathrm{mg} / \mathrm{L})$ and ciprofloxacin
Outcome of the expert discussion following the third case of Neisseria meningitidis in a nursery, England, June 2013

Information on the outbreak strain $N$. meningitidis group B, serotype 4 , serosubtype P1.NT/NT/6

- Sero-subtyping markers showed that this is an extremely rare strain.

- Since 2007, there have only been two other cases of similar sero-subtypes in the United Kingdom.

- This subtype has not been identified in carriage studies; carriage rate and patterns of carriage are therefore unknown.

Agreed actions

- Offer chemoprophylaxis to all staff and children of Nursery 1.

- Use ciprofloxacin for chemoprophylaxis. Ciprofloxacin had already been used in the initial response to Cases 1 and 2, however:

- Ciprofloxacin is more effective at eradicating nasopharyngeal carriage than rifampicin;

- There is no microbiological evidence that the first two cases were resistant to ciprofloxacin.

- Given that this is a rare subtype for which carriage rates, virulence and the effectiveness of chemoprophylaxis are currently unknown, undertake swabbing as part of the operational public health response to inform action: - Swabbing should not delay chemoprophylaxis;

- It is recognised that swabbing does not always identify carriage.

$(\mathrm{MIC}=0.004 \mathrm{mg} / \mathrm{L})$. Routine typing was performed in accordance with Gray et al. [15].

Nine days after the first case was admitted, a case of probable meningococcal disease in a two year-old child (Case 2) from the same nursery as Case 1 was notified. The child presented with a non-blanching rash, lethargy, vomiting and fever. Given that Case 1 was caused by a strain not preventable by the vaccines available at the time, chemoprophylaxis (and not vaccine) was offered to a defined group of staff and children at Nursery 1. UK guidance recommends that the target group should be 'discrete' and 'make sense to staff and parents' [9]. In this case, we offered chemoprophylaxis to children and staff associated with two of the four classes who were considered to be close contacts. Close contacts were defined as any child or adult at the nursery who spent between four and eight hours in the same room as the two cases. In total, 38 children and nine staff were offered chemoprophylaxis the day after the second case was admitted. All children and staff collected a letter from the nursery to take to their general practitioner, which advised that they had been a contact of cases of meningococcal disease and chemoprophylaxis was recommended. Self-reported uptake of chemoprophylaxis was $100 \%$.

The causative organism for Case 2 was confirmed by both EDTA PCR and culture as meningococcus serogroup $B$ and subsequently as the same PorA subtype 
as in Case 1, P1.18-4, 25, 38-1. The isolate from Case 2 had reduced susceptibility (intermediate) to penicillin $(M I C=0.06 \mathrm{mg} / \mathrm{L})$ but was susceptible to cefotaxime $(\mathrm{MIC}=0.004 \mathrm{mg} / \mathrm{L})$, rifampicin $(\mathrm{MIC}=0.004 \mathrm{mg} / \mathrm{L})$ and ciprofloxacin (MIC $=0.004 \mathrm{mg} / \mathrm{L})$.

In June (over 18 weeks after the first case was admitted to hospital), the health protection team was notified of a probable case of meningococcal septicaemia in a two year-old child (Case 3) who attended the same nursery class as Cases 1 and 2 and who had received chemoprophylaxis in February. While awaiting typing results, given the time between the cases and as per UK guidance, the initial public health response was based on the assumption that the latest case was likely to be unrelated to the previous cases. The case was subsequently confirmed as meningococcus serogroup $B$ by PCR alone and later as subtype PorA P1.18-4, 25, 38-1. All cases made a good recovery with no longterm sequelae.

\section{Public health investigation}

Following confirmation 10 days after the third case was admitted, that the third case in Nursery 1 (Case 3) was infected with the same rare subtype by PorA sequencing, the outbreak control team consulted national experts at the Centre for Infectious Disease Surveillance and Control at Colindale and the Meningococcal Reference Unit. The Box summarises information on the subtype and actions agreed at the meeting with the experts, which was held the day after typing results were available for the third case in Nursery 1.

\section{Identifying potentially linked cases}

In an attempt to identify others affected by the outbreak strain, molecular PorA subtyping results were sought from all cases of meningococcal disease where typing had been undertaken from the local area (Warwickshire and Coventry) notified between January and July 2013.

\section{Further epidemiological investigation}

Telephone interviews were conducted to identify potential links between these cases. Two questionnaires were developed and both were piloted to ensure ease of understanding and use. The first was used to capture information from adult cases and the second was used to obtain information from parents if the case was a child. Questions covered regular activities, events, gatherings and links with local nurseries, schools and mother and toddler groups as well as activities in the week before the case became unwell.

\section{Assessing carriage in Nursery 1}

To support the epidemiological investigation, throat swabs were requested from all children and staff of Nursery 1 prior to prophylaxis. Swabbing took place the day after the meeting with the experts. Parental consent was secured for swabbing of children. Parents and staff were informed that individual results would not be disclosed, in line with usual practice. Ethical approval for throat swabbing was not obtained as it was part of the outbreak response.

\section{Additional molecular investigations}

The Meningitis Research Foundation Meningococcus Genome Library (MGL; http://www.meningitis.org/ genome-library) contains genomic sequence data for all IMD case isolates from the epidemiological years $2010 / 11$ to $2012 / 13$. Genomic sequence data for the three outbreak isolates became available via the MGL in February 2014. Full finetype data (Group: PorA: FetA: multilocus sequence type (ST) and clonal complex $(\mathrm{mL}))$ was queried against the library and the library's

\section{FIGURE 1}

Timeline of meningitis cases, nursery outbreak in England, February to June 2013 (n=5)

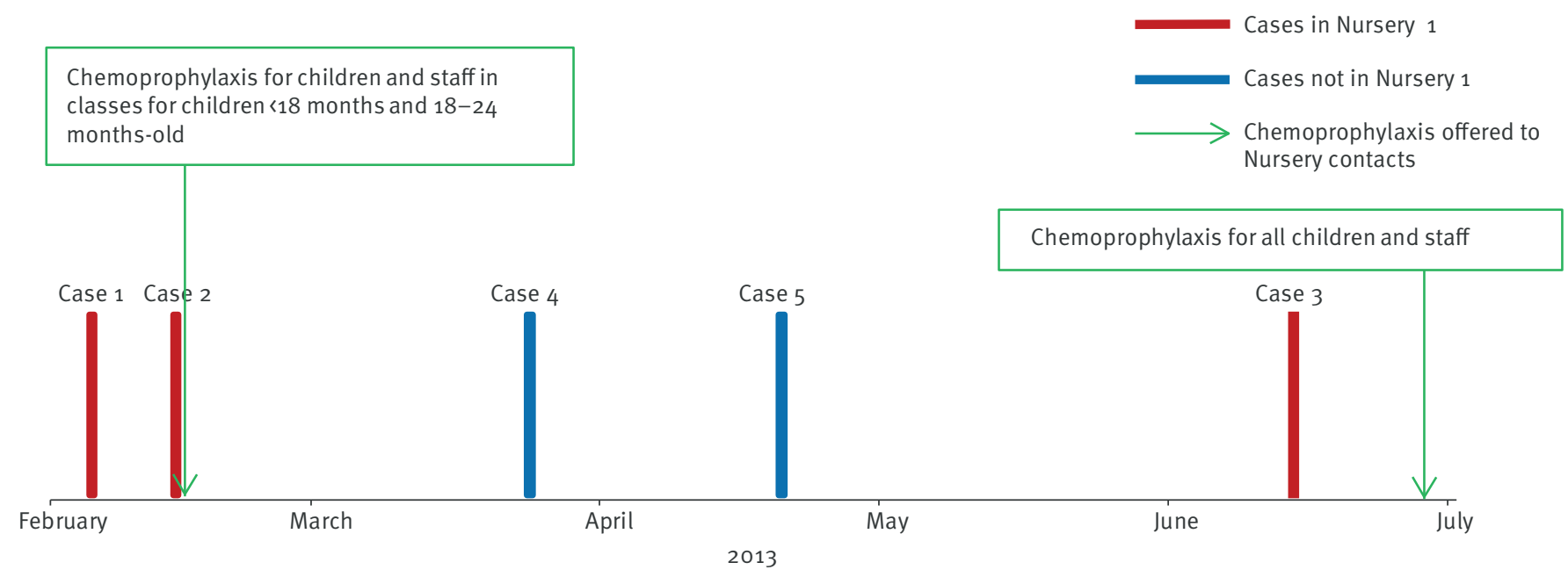


Results of throat swabs taken at the nursery in context with the meningitis outbreak in England, February to June 2013 $(n=69)$

\begin{tabular}{|l|c|c|c|c|c|c|} 
& $\begin{array}{c}\text { Number of } \\
\text { individuals }\end{array}$ & $\begin{array}{c}\text { Individuals } \\
\text { swabbed } \\
\mathrm{n}(\%)\end{array}$ & $\begin{array}{c}\text { Individuals swabbed who had } \\
\text { received prophylaxis in February } \\
\mathrm{n}(\%)\end{array}$ & $\begin{array}{c}\text { Swabs } \\
\text { processed by } \\
\text { laboratory } \\
\mathrm{n}(\%)\end{array}$ & $\begin{array}{c}\text { Carriage } \\
\mathrm{n}(\%)^{\mathrm{b}}\end{array}$ & $\begin{array}{c}\text { Carriage of } \\
\text { outbreak strain } \\
\%\end{array}$ \\
\hline Staff & 16 & $15(94)$ & $7(47)$ & $14(88)$ & $0(0.0)$ & 0 \\
\hline Children & 95 & $54(57)$ & $12(22)$ & $53(56)$ & $1(1.9)^{\mathrm{c}}$ & 0 \\
\hline Total & 111 & $69(62)$ & $19(28)$ & $67(60)$ & $1(1.5)$ & 0 \\
\hline
\end{tabular}

Two swabs were not processed because of unclear labelling.

Carriage was estimated using swab results positive for meningococcus. The denominator used to calculate carriage was the number of swabs taken at the nursery which were processed by the laboratory.

This child was not given antibiotic prophylaxis in February 2013.

BIGSdb genome comparator tool was used to perform partial core-genome comparisons (1,546 loci) between these and related isolates both in the MGL (comprising all English, Welsh and Northern Irish IMD isolates from July 2010 to June 2013) and the wider PubMLST Neisseria database (http://pubmlst.org/neisseria/). NeighborNet phylogenetic networks were produced by applying SplitsTree4 (version 4.12.8) [16] to the distance matrices generated. For two culture negative cases (Cases 3 and 4), non-culture characterisation of sub-variants of the meningococcal factor $\mathrm{H}$ binding protein (fHbp) was performed according to Clark et al. [17].

\section{Results}

\section{Identifying potentially linked cases}

Subsequently, after PorA sequence typing of six geographically and temporally associated group B cases identified by the health protection team (i.e. cases notified within the six months before mid-June 2013), the reference laboratory notified the health protection team of two additional cases with the same PorA subtype. The first was a person in their 6os (Case 4) identified by PCR alone (onset March 2013) whose grandchild attended Nursery 1 and had been offered prophylaxis in February. The second was a two year-old child (Case 5) from an unconnected nursery (Nursery 2) who became unwell with a non-blanching petechial rash in April 2013 and was confirmed by both PCR and culture. The isolate from Case 5 was also of reduced (intermediate) susceptibility to penicillin ( $\mathrm{MIC}=0.06$ $\mathrm{mg} / \mathrm{L}$ ) but susceptible to cefotaxime (MIC $=0.004$ $\mathrm{mg} / \mathrm{L})$, rifampicin (MIC $=0.004 \mathrm{mg} / \mathrm{L}$ ) and ciprofloxacin $(\mathrm{MIC}=0.004 \mathrm{mg} / \mathrm{L})$. Nursery 2 is situated $12.1 \mathrm{~km}$ from Nursery 1. Four further PCR-confirmed group B cases in children investigated in the area in February to June yielded PorA subtypes P1.22, 9, 35-1, P1.7-2, 4, 37 and two cases with $P_{1} 19,15,36$, all quite distinct to the outbreak strain. Figure 1 shows the timeline of the five $\mathrm{P}_{1.18-4}, 25,38-1$ cases and public health actions taken.
Further epidemiological investigation Interviewing did not identify social links among cases except Nursery 1 for four of the five cases.

\section{Assessing carriage within Nursery 1}

In order to test for meningococcal carriage, throat swabs were collected from 15 of 16 staff and 54 of 95 children (Table). Swabs were taken from all staff and children who consented and were able to attend the nursery on the day of swabbing. Swabbing was not repeated so as not to delay chemoprophylaxis. It was not possible to collect throat swabs from all children if parental consent was not obtained or the children did not tolerate the procedure. One member of staff was not swabbed because they were abroad at the time of swabbing. The swabs were screened for any query Neisseria species. Neisseria lactamica was not tested for. A meningococcus was isolated from one child who had not been identified as eligible for the initial prophylaxis, and was phenotypically characterised as group NG, serotype 21 and sero-subtype (NT / NT / NT) with PorA $\mathrm{P}_{1.18-1,30-9,38}$. The isolate was resistant to penicillin ( $\mathrm{MIC}=0.50 \mathrm{mg} / \mathrm{L}$ ) but susceptible to cefotaxime $(\mathrm{MIC}=0.008 \mathrm{mg} / \mathrm{L})$, rifampicin $(\mathrm{MIC}=0.016 \mathrm{mg} / \mathrm{L})$ and ciprofloxacin $(\mathrm{MIC}=0.008 \mathrm{mg} / \mathrm{L})$. The outbreak strain was not identified from any swab taken.

\section{Additional molecular investigations}

The three available outbreak isolates (Cases 1, 2 and

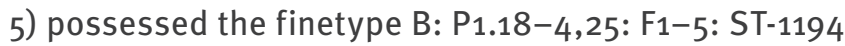
(cc41/44), which was unique within the PubMLST Neisseria database (including the MGL). A partial core genome comparison among all cc41/44 genomes on the PubMLST Neisseria database $(n=484$, of which 334 were also in the MGL; accessed 22 May 2014) indicated that the three outbreak isolates formed part of a discrete cluster of 85 isolates, all of which were isolated in the UK or Ireland. In a further partial core genome comparison, Cases 1 and 2 differed from one another at 11 of 1,250 variable loci and from Case 3 at 20 and 22 loci, respectively. When treating putative recombinations (differences among two or more neighbouring loci) as single differences, the corresponding figures 


\section{FIGURE 2}

Phylogenetic network analyses of Neisseria meningitidis outbreak isolates and closely related isolates in the PubMLST Neisseria database and Meningococcus Genome Library

A

B
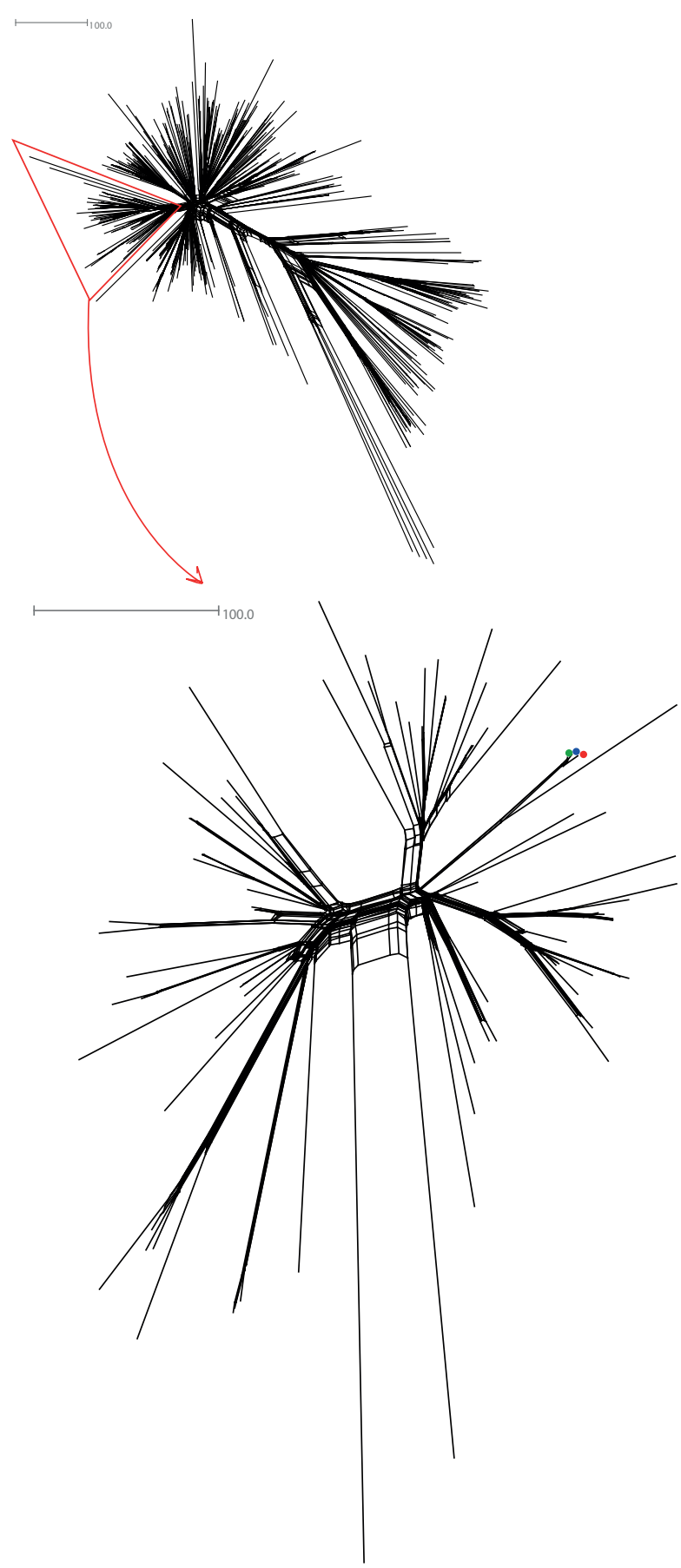

Network $A$ is based on a partial core genome comparison of all cc41/44 isolates on the PubMLST Neisseria database including those of the Meningococcus Genome Library (MGL) representing all English, Welsh and Northern Irish isolates from July 2010 to June 2013 (inclusive). The red triangle highlights a discrete cluster of 85 isolates including those of outbreak Cases 1, 2 and 5 . Network B is based on a separate partial core genome comparison of the cluster of 85 isolates in which isolates from Cases 1, 2 and 5 are highlighted green, blue and red, respectively. Scale bars indicate the number of differences among 1,546 loci compared. were 6,10 and 9 loci, respectively. By contrast, the average distance between case one and the remaining 82 closely related isolates (not adjusting for putative recombinations) was 256 differences (standard deviation: 66, range: $142-502$ ) (Figure 2 ).

In terms of $\mathrm{fHbp}$, the isolates from Cases 1, 2 and 3 all possessed alleles for peptide 9, the isolate from Case 4 possessed an allele for peptide 30 , and the isolate from Case 5 an allele for peptide 4 .

\section{Discussion}

The health protection team identified three plausible explanations for the outbreak. Firstly, the initial prophylaxis at Nursery 1 may not have eradicated carriage within the nursery. It is unlikely that prophylaxis failed to eradicate carriage for the following reasons:

(i) Given the length of time between the cases at Nursery 1 and the negative swabs from nursery staff and children who were not given prophylaxis initially, and given that meningococcal carriage is low in this age group of children [18], it is unlikely that the initial prophylaxis did not include enough people and the outbreak strain was reintroduced to the affected class from within the nursery. In addition, the existence of a case in the wider community without a link to Nursery 1 makes this unlikely. However, it should be noted that not all children and staff at Nursery 1 were swabbed, and therefore a carrier may have been missed.

(ii) Uptake of prophylaxis was assumed to be good since all parents and staff collected the antibiotic prescriptions and reported taking chemoprophylaxis.

(iii) It is unlikely that the organism was resistant to the antibiotic used for initial prophylaxis given that resistance to ciprofloxacin is unusual [19] and swab results were negative for the outbreak strain among those who received prophylaxis, although not all were tested. In addition, the isolates from all three culture-confirmed cases (Cases 1, 2 and 5) were ciprofloxacin-sensitive.

Secondly, the notified cases could have been the beginning of a community outbreak. Subtyping results of recent local cases of meningococcal infection identified no additional cases with the outbreak strain and no subsequent cases (as of 25 October 2013).

Finally, there may be another undefined social network connecting the cases. This explanation is plausible because the initial two cases occurred within nine days, while the final case occurred more than 18 weeks later. Transmission could occur via one common contact or via social networks in the wider community. We tried unsuccessfully to identify social links through interviewing. However, an unidentified network is still the most plausible explanation given the known links in time and place and the person characteristics. 
The partial core genome comparisons confirmed that the isolates from Cases 1, 2 and 5 were very closely related to one another but were relatively distant from any other English, Welsh or Northern Irish isolates collected between July 2010 and June 2013. This strongly supports earlier conclusions, based on spatiotemporal evidence and routine typing data, that Cases 1 and 2 constituted a genuine outbreak and that the outbreak was protracted, extending to Case 5 in the wider community. They also confirmed the absence of additional, but previously unidentified, culture-confirmed cases dating back to June 2010 .

The rarity of PorA P1.18-4,25 (unique within the MGL to the three outbreak isolates) indicated the inclusion of the non-culture (confirmed by PCR only) Cases 3 and 4 in the outbreak investigation. The now routine typing target fhbp, however, differed between Case 4 (peptide 30), Case 5 (peptide 4) and Cases 1 to 3 (encoding peptide 9). The difference between Case 5 and Cases 1 and 2, given the close relationship identified through core genome comparisons, is likely to have arisen through recombination (the alleles were substantially different) which is not uncommon in meningococci [20]. This highlights the potential of a single antigen gene to, somewhat misleadingly, distinguish very closely related isolates. Thus the differing $\mathrm{fHbp}$ variant did not preclude the inclusion of Case 4, especially given the spatiotemporal link and the rare PorA variant. Routine real-time whole genome sequence analysis of case isolates and, eventually, non-culture specimens, may help us to confirm or rule out such uncertain, protracted outbreaks in a suitable timescale to influence management. At present, isolates are added to the MGL in batches at the end of the epidemiological year. Nonetheless, the detailed molecular characterisation that became available later did not support a different approach to that taken by the outbreak management team.

cc41/44 is a diverse clonal complex that has been a major cause of IMD throughout Europe and further afield for over a decade [21]. The sequence type ST-1194 first occurred in the PubMLST Neisseria database in 1999 when it was assigned to an Irish isolate. Further ST-1194 isolates have since been added representing ten European countries (Czech Republic $(n=6)$, France $(n=2)$, Germany $(n=10)$, Ireland $(n=11)$, Italy $(n=3)$, the Netherlands $(n=1)$, Slovenia $(n=1)$, Spain $(n=1)$, Sweden $(n=1)$ and the UK $(n=38))$, as well as the US $(n=4)$ and South Africa $(n=1)$, indicative of widespread distribution of the strain. The corresponding isolates were associated with 14 different PorA subtypes, of which $\mathrm{P}_{1.18-4}$, 25, however, was only found among the outbreak isolates described herein. Similarly, in the phylogenetic analyses, these occupied an isolated sub-lineage in which they diverged from a single Irish isolate (isolated 2011) possessing the relatively common PorA P1.18-1,3. Thus, while PorA may serve to highlight potentially related cases occurring subsequently in the UK or further afield, only genomic analyses can confirm this.

There is now growing evidence that outbreaks of meningococcus group $B$ are different from meningococcus $C$ outbreaks as they may be prolonged [13]. Our outbreak fits this pattern and highlights that guidance that is predominantly based on experience of meningococcus $C$ outbreaks may not always be appropriate for serogroup B outbreaks. However, in response to the recent licensure of a vaccine targeting capsular group $B$ meningococci, new recommendations for preventing secondary cases have been prepared in the UK [22].

The availability of meningococcal typing information led to a substantially different public health response to the situation as cases, other than the initial two, would have been treated as sporadic. The important lesson learnt was therefore that subtyping results should be reviewed early in incidents with a potential epidemiological link involving the same meningococcal serogroup, even when cases occur outside the recognised time limits used to define a cluster. Since the occurrence of this cluster, new guidance in the UK has been published which recommends that for clusters involving two or more confirmed/probable cases who are or could be infected with the same capsular group $B$ strain, vaccination using the recently licensed vaccine should be offered to the same group that would receive antibiotic chemoprophylaxis [22]. In this instance, vaccine would have been offered after the second case in Nursery 1 and may have prevented subsequent cases.

\section{Conclusion}

In the investigation of our outbreak, we did not identify any other link among cases than attendance at Nursery 1. However, the length of time between illness onset and interview may have affected the respondents' recollection of details that could have identified potential links. Therefore, to identify potential links among social networks we suggest that a standard questionnaire should be administered to all cases as soon as an outbreak is suspected. Use of research from social science around networks and social interaction could also help develop robust methods for identifying social links between cases.

\section{Acknowledgements}

The authors would like to acknowledge the rest of the outbreak control team, namely Catherine Alves and Katherine Martin in the local health protection team, Michael Caley in the public health department at Warwickshire County Council, and colleagues at the Centre for Infectious Disease Surveillance and Control (Colindale), Public Health England and the Meningococcal Reference Unit, Public Health England (Shamez Ladhani, Sema Mandal, Ed Kaczmarski).

This publication made use of the Meningitis Research Foundation Meningococcus Genome Library (http://www. meningitis.org/research/genome) developed by Public Health England, the Wellcome Trust Sanger Institute and the University of Oxford as a collaboration. The project is funded by Meningitis Research Foundation. 


\section{Conflict of interest}

None declared.

\section{Authors' contributions}

CC, RG, KN, MT, ML, SG and TF were members of the outbreak team. CC and TF prepared the initial manuscript. CC, RG, JH, $\mathrm{KN}, \mathrm{MT}, \mathrm{ML}, \mathrm{SG}, J \mathrm{~L}, \mathrm{AC}, \mathrm{SC}$ and TF contributed to the subsequent editorial revisions. SG, JL, AC and SC performed laboratory investigations and genomic analyses.

\section{References}

1. de Assis Aquino Gondim F. Meningococcal meningitis. Background. New York: Medscape; December 2013. Available from: http://emedicine.medscape.com/ article/1165557-overview\#a0101

2. Public Health England (PHE). Meningococcal: the green book, chapter 22. London: PHE; March 2013. Available from: https://www.gov.uk/government/publications/ meningococcal-the-green-book-chapter-22

3. Military Vaccine Agency - Vaccine Healthcare Centers Network. Questions and Answers. Meningococcal - the disease. Falls Church: Defense Health Headquarters. May 2014. Available from: https://www.vaccines.mil/Questions_and_Answers/ Disease/Meningococcal/The_Disease

4. Centers for Disease Control and Prevention (CDC). Active bacterial core surveillance ( $A B C s$ ) report emerging infections program network Neisseria meningitidis, 2012. Atlanta: CDC; December 2013. Available from: http://www.cdc.gov/abcs/ reports-findings/survreports/mening12.pdf

5. European Centre for Disease Prevention and Control (ECDC). Surveillance of invasive bacterial diseases in Europe, 2011. Stockholm: ECDC; December 2013. Available from: http:// www.ecdc.europa.eu/en/publications/Publications/invasivebacterial-diseases-surveillance-2011.pdf

6. Public Health England (PHE). Health protection - collection. Meningococcal disease: guidance, data and analysis. London: PHE. [Accessed: January 29, 2014]. Available from: http:// www.hpa.org.uk/Topics/InfectiousDiseases/InfectionsAZ/ MeningococcalDisease/EpidemiologicalData/

7. European Centre for Disease Prevention and Control (ECDC). Public health management of sporadic cases of invasive meningococcal disease and their contacts. Stockholm: ECDC; October 2010. Available from: http://www.ecdc.europa.eu/en/ publications/Publications/1010_GUI_Meningococcal_guidance. pdf

8. Hastings L, Stuart J, Andrews N, Begg N. A retrospective survey of clusters of meningococcal disease in England and Wales, 1993 to 1995: estimated risks of further cases in household and educational settings. Commun Dis Rep CDR Rev. 1997;7(13):R195-200. PMID:9447784

9. Health Protection Agency. Guidance for public health management of meningococcal disease in the UK. London: HPA; March 2012. Available from: https://www.gov.uk/ government/uploads/system/uploads/attachment data/ file/322008/Guidance_for_management_of_meningococcal_ disease_pdf.pdf

10. Davison KL, Andrews N, White IM, Ramsay ME, Crowcroft NS, Rushdy AA, et al. Clusters of meningococcal disease in school and preschool settings in England and Wales: what is the risk? Arch Dis Child. 2004;89(3):256-6o. http://dx.doi.org/10.1136/ adc.2003.031369 PMID:14977705

11. Zangwill KM, Schuchat A, Riedo FX, Pinner RW, Koo DT, Reeves MW, et al. School-based clusters of meningococcal disease in the United States. Descriptive epidemiology and a case-control analysis. JAMA. 1997;277(5):389-95. http://dx.doi.org/10.1001/ jama.1997.03540290041030 PMID:9010171

12. Acheson P, Barron R, Borrow R, Gray S, Marodi C, Ramsay M, et al. A cluster of four cases of meningococcal disease in a single nuclear family. Arch Dis Child. 2012;97(3):248-9. http://dx.doi. org/10.1136/archdischild-2011-301074 PMID:22247241

13. Mandal S, Wu HM, MacNeil JR, Machesky K, Garcia J, Plikaytis $\mathrm{BD}$, et al. Prolonged university outbreak of meningococcal disease associated with a serogroup $B$ strain rarely seen in the United States. Clin Infect Dis. 2013;57(3):344-8. http://dx.doi. org/10.1093/cid/cit243 PMID:23595832

14. Ashton $P$, Dallman T, Jenkins $C$, Perry N, Byrne L, Elson R, et al. The impact of whole-genome sequencing on the detection and investigation of outbreaks of Escherichia coli $0157: \mathrm{H}_{7}$ in the UK. 23rd European Congress of Clinical Microbiology and Infectious Diseases (ESCMID),Berlin, 27-30 April 2013.

15. Gray SJ, Trotter CL, Ramsay ME, Guiver M, Fox AJ, Borrow R, et al. Epidemiology of meningococcal disease in England and Wales 1993/94 to 2003/04: contribution and experiences of the Meningococcal Reference Unit. J Med Microbiol. 2006;55(Pt 7):887-96. http://dx.doi.org/10.1099/jmm.0.46288-0 PMID:16772416

16. Huson DH. SplitsTree: analyzing and visualizing evolutionary data. Bioinformatics. 1998;14(1):68-73. http://dx.doi. org/10.1093/bioinformatics/14.1.68 PMID:9520503

17. Clark SA, Lucidarme J, Newbold LS, Borrow R. Genotypic analysis of meningococcal factor h-binding protein from non-culture clinical specimens. PLoS ONE. 2014;9(2):e89921. http://dx.doi.org/10.1371/journal.pone.0089921 PMID:24587125

18. Cartwright KA, Stuart JM, Jones DM, Noah ND. The Stonehouse survey: nasopharyngeal carriage of meningococci and Neisseria lactamica. Epidemiol Infect. 1987;99(3):591-601. http://dx.doi.org/10.1017/S0950268800066449 PMID:3123263

19. Kaczmarski EB, Gray SJ, Carr AD, Mallard RH. Antimicrobial agent susceptibility of clinical Neisseria meningitidis isolates from England and Wales. Abstracts of the Thirtyninth Interscience Conference on Antimicrobial Agents and Chemotherapy, San Francisco, 26-29 September 1999.

20. Buckee CO, Gupta S, Kriz P, Maiden MC, Jolley KA. Long-term evolution of antigen repertoires among carried meningococci. Proc Biol Sci. 2010;277(1688):1635-41. http://dx.doi. org/10.1098/rspb.2009.2033 PMID:20129981

21. Harrison LH, Trotter CL, Ramsay ME. Global epidemiology of meningococcal disease. Vaccine. 2009;27(Suppl 2):B51 63. http://dx.doi.org/10.1016/j.vaccine.2009.04.063 PMID:19477562

22. Ladhani S, Cordery R, Mandal S, Christensen H, Campbell $H$, Borrow R, et al. Preventing secondary cases of invasive meningococcal capsular group B (MenB) disease: benefits of offering vaccination in addition to antibiotics chemoprophylaxis to close contacts of cases in the household, educational setting, clusters and wider community. London: Public Health England; April 2014. Available from: https:// www.gov.uk/government/uploads/system/uploads/ attachment_data/file/328835/Invasive meningococcus secondary_case_prevention_April_2014.pdf 